\title{
高温对汽车灰铸铁制动盘热疲劳裂纹 萌生寿命的影响
}

\author{
刘禹 1,2 单颖春 1,2 刘献栋 ${ }^{1,2}$ 何 田 1,2
}

(1. 北京航空航天大学交通科学与工程学院 北京 100191 ;

2. 北京航空航天大学新能源汽车高效动力传动与系统控制北京市重点实验室 北京 100191)

摘要: 汽车制动盘的工作温度高、易产生热疲劳, 其性能直接影响行车安全, 对高温下制动盘的热疲劳裂纹萌生寿命的研究 十分必要。首先研究取自汽车制动盘上的灰铸铁 HT200 试样在 $500{ }^{\circ} \mathrm{C}$ 下单调拉伸与压缩的性能, 对应力一应变曲线进行分 析, 得到其力学性能参数; 接着基于这些参数, 对初始温度为 $400{ }^{\circ} \mathrm{C}$ 时的制动盘在单次紧急制动工况下进行热-结构耦合仿 真分析, 得到制动盘的温度场和应力场分布; 最后利用应变疲劳的方法根据 Miner 线性累积损伤理论研究 $500{ }^{\circ} \mathrm{C}$ 下灰铸铁 HT200 的塑性特性对制动盘热疲劳裂纹萌生寿命的影响。研究结果表明: 制动过程中的热应力远大于机械应力, 是产生疲劳 裂纹的主要原因; 高温下制动盘材料 HT200 的塑性特性对制动盘热疲劳裂纹萌生寿命的影响很大, 在研究制动盘裂纹萌生寿 命时需考虑高温下塑性特性对寿命的影响。利用制动盘在高温制动过程中的周向应变并考虑高温下材料的塑性特性计算热疲 劳裂纹萌生寿命, 为制动盘热疲劳寿命的评价打下基础。

关键词: 灰铸铁 HT200; 拉伸/压缩试验; 热-结构耦合; 热疲劳裂纹萌生寿命

中图分类号: U463

\section{Effect of High Temperature on Thermal Fatigue Crack Initiation Life of Automotive Gray Cast Iron Brake Disc}

\author{
LIU Yu ${ }^{1,2}$ SHAN Yingchun ${ }^{1,2}$ LIU Xiandong ${ }^{1,2}$ HE Tian ${ }^{1,2}$ \\ (1. School of Transportation Science and Engineering, Beihang University, Beijing 100191; \\ 2. Beijing Key Laboratory for High-efficient Power Transmission and System Control \\ of New Energy Resource Vehicle, Beihang University, Beijing 100191)
}

\begin{abstract}
The brake disc of a vehicle often works at high temperature, and its damage caused by thermal fatigue easily takes place. So the performance of the vehicle brake disc is directly related to the safety of driving, and it is very necessary to study the thermal fatigue crack initiation life of the brake disc at high temperature. The monotonic tensile and compressive tests of gray cast iron HT200 sample for vehicle brake discs are carried out at $500{ }^{\circ} \mathrm{C}$, and the stress-strain curves are analyzed to obtain mechanical properties at $500{ }^{\circ} \mathrm{C}$; then based on these parameters, the finite element software ABAQUS is used to establish the thermal-structure coupled finite element model of the vehicle brake disc with the initial temperature of $400{ }^{\circ} \mathrm{C}$, and the temperature fields and stress fields on the brake disc for emergency braking condition are analyzed. Finally, the effect of the plastic property of HT200 on the thermal fatigue crack initiation life of the brake disc on the $500{ }^{\circ} \mathrm{C}$ is studied by using strain fatigue method according to the Miner linear cumulative damage theory. The results show that the thermal stress during braking is far greater than the mechanical stress, which is the main cause of fatigue cracks; the plastic property of gray cast iron HT200 of brake disc has a great influence on the thermal fatigue crack initiation life of the brake disc. The effect of plastic property on the life of the brake disc should be introduced in the study of the crack initiation life of the brake disc. The thermal fatigue crack initiation life of brake disc is calculated by using the circumferential strain of brake disc during braking at high temperature and considering the plastic properties of material at high temperature, which lays a foundation for the evaluation of thermal fatigue life of brake disc.
\end{abstract}

Key words: gray cast iron HT200; tensile and compressive test; thermal-structure coupled; thermal fatigue crack initiation life 


\section{0 前言}

铸铁材料因其铸造性能好、生产成本低、导热 系数高等优点, 广泛用作汽车制动盘材料。汽车制 动时, 制动祄块与制动盘之间产生的摩擦将汽车动 能转化为热能, 因此使用中制动盘的温度变化范围 很大。在冬季, 不制动时制动盘的温度常在零下几 十度, 但经过数次制动后, 制动盘表面温度常达到 $500{ }^{\circ} \mathrm{C}$ 左右 ${ }^{[1]}$ 。汽车制动盘生产企业的调查显示, $400 \sim 500{ }^{\circ} \mathrm{C}$ 是制动盘常见工作温度。在如此恶劣的 工作环境下，要保证制动系统正常工作，就必须对 制动盘的热疲劳裂纹萌生寿命, 尤其是高温下疲劳 寿命, 进行深入研究。

近年来, 针对制动盘的热疲劳裂纹萌生寿命的研 究主要是利用低周疲劳方法进行的。GIGAN ${ }^{[2]}$ 研究汽 车制动盘通风槽的布置形式对制动盘热疲劳寿命的 影响, 首先利用有限元方法得到连续制动过程中的周 向应力分布, 接着利用低周疲劳方法计算出热疲劳寿 命。结果表明呈一定角度布置的通风槽比直线布置的 通风槽的寿命提高 $50 \%$ 。KAKANDAR 等 ${ }^{[3]}$ 利用应变 疲劳的方法研究了通风式制动盘的几何尺寸对疲劳 寿命的影响, 结果表明通风槽的厚度和有效偏移长度 对疲劳寿命的影响最大。

除汽车外, 铁道车辆也使用盘式制动器进行制 动, 并且该领域技术人员对高速列车制动盘的热疲劳 裂纹萌生寿命也进行了大量研究。ZHANG 等 ${ }^{[4]}$ 对利 用顺序热-力耦合的方法对高速列车制动盘的温度场 和应力场进行了仿真分析, 并建立了制动盘的几种缺 陷模型, 并通过比较热应力分析了缺陷的位置、深度、 尺寸等参数对裂纹萌生的影响, 结果表明, 制动盘表 面的塑性变形是由压应力引起的, 裂纹的萌生与缺陷 的形状和尺寸关系较大。SONSINO 等 ${ }^{[5]}$ 以球墨铸铁 EN-GJS-400-15 高速铁路制动盘为研究对象, 在制动 台架上进行初速度为 $200 \mathrm{~km} / \mathrm{h} 、 160 \mathrm{~km} / \mathrm{h} 、 90 \mathrm{~km} / \mathrm{h}$ 的紧急制动至停止, 并利用等效应变幅计算疲劳寿 命, 最后对制动盘的结构进行优化, 结果表明增大通 风槽端部半径, 改变盘与螺栓搭接件的截面积可以提 高疲劳寿命。KIM 等 ${ }^{[6]}$ 提出了一种估算疲劳寿命的方 法, 首先研究了高速列车制动盘 HT250 材料在室温 至 $300{ }^{\circ} \mathrm{C}$ 时的循环拉压特性, 接着利用有限元方法 计算制动过程中制动盘的温度场和周向应力场分布, 最终利用应力疲劳的方法计算疲劳寿命。结果表明 HT250 在室温至 $300{ }^{\circ} \mathrm{C}$ 的力学特性变化不明显, 周 向应力与温度呈近似线性的关系。除了常用的灰铸铁 材料制动盘, 还有学者对复合材料制动盘的寿命进行
研究。王文静等 ${ }^{[7]}$ 以高速列车制动盘 $\mathrm{SiCp}$ A356 材料 为研究对象, 对材料进行室温与高温下的单调拉伸和 循环拉伸试验得到材料本构关系模型，接着对制动盘 在制动过程中的温度场和应力场进行研究, 最后给出 制动盘的疲劳-蠕变损伤寿命预测模型。结果表明 SiCp_A356 复合材料制动盘的热疲劳寿命必须考虑 蠕变损伤的影响。

但是, 经检索文献得知, 已有发表文献在研究 热疲劳裂纹萌生寿命时，均将灰铸铁在高温下视为 线弹性材料，未考虑到高温下灰铸铁的塑性特征， 而且未对仿真得到的应力做拉伸或者压缩的判断, 在计算应变疲劳寿命时仅利用最大应变幅来计算, 也未考虑各个应变循环对寿命的影响。但实际情况 是灰铸铁在高温下的抗压强度约是抗拉强度的两 倍, 弹性模量与压缩模量相差近 $10 \%$, 因此不对应 力做拉伸或者压缩的判断而直接使用等效应力来计 算寿命必会导致寿命计算产生误差。另外，根据工 程经验，制动盘的热疲劳属于低周疲劳，所以利用 应变疲劳的方法计算寿命更符合工程实际，且由于 温度在制动盘表面呈周期性变化，若只考虑最大应 变幅计算寿命, 即忽略了每一次温度循环所产生的 热应力对制动盘造成的损伤, 计算得到的寿命将比 实际情况偏大, 不利于制动盘在设计时的寿命评估。

针对以上问题，考虑到汽车进行多次制动时，制 动盘常处在 $400 \sim 500{ }^{\circ} \mathrm{C}$ 的高温环境，因此假设制动 盘的初始温度为 $400{ }^{\circ} \mathrm{C}$ 。考虑到再次制动后温度在 $500{ }^{\circ} \mathrm{C}$ 左右, 本文首先对 $\mathrm{HT} 200$ 在 $500{ }^{\circ} \mathrm{C}$ 下进行单 调拉伸与单调压缩试验, 获得其力学性能参数, 之后 基于这些参数进行紧急制动工况下的制动盘热-结构 耦合仿真分析, 得到温度场与等效应力场, 并利用应 变疲劳的方法根据 Miner 线性累积损伤理论计算制动 盘的热疲劳裂纹萌生寿命，最后分析高温下 HT200 的塑性对热疲劳裂纹萌生寿命的影响。

\section{1 试件的试验测试及结果分析}

按《金属材料高温拉伸试验方法 GB/T 4338$1995\rangle^{[8]}$ 《HB7571-1997 金属高温压缩试验方法》 ${ }^{[9]}$ 分别进行 $500{ }^{\circ} \mathrm{C}$ 下的单调拉伸试验与单调压缩试 验。每次试验, 分别对三个有效试样进行重复试验。 用于测试材料抗拉强度和弹性模量的试样如图 1a 所示, 抗压强度的测试试样如图 $1 \mathrm{~b}$ 所示, 压缩模量 的测试试样如图 1c 所示。试验在 MTS810 伺服液压 疲劳试验机上进行。500 ${ }^{\circ} \mathrm{C}$ 单调拉伸试验加载速率 为 $0.12 \mathrm{~mm} / \mathrm{min}$, 单调压缩试验加载速率为 0.22 $\mathrm{mm} / \mathrm{min}$ 。 


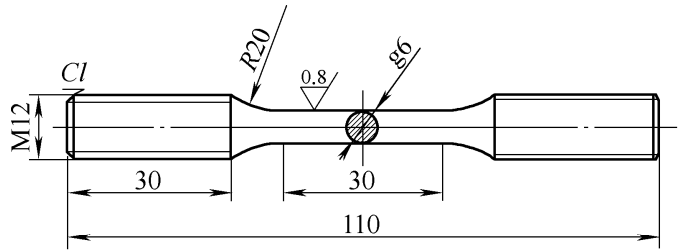

(a)
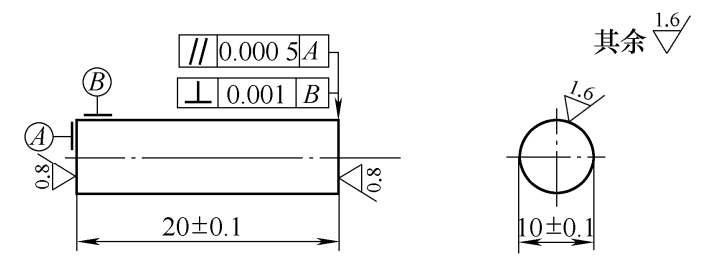

(b)

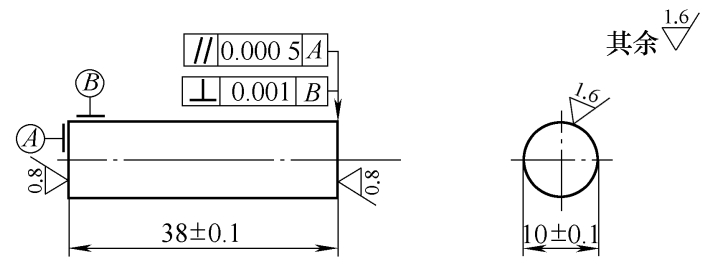

(c)

图 1 试验试样

灰铸铁 HT200 在 $500{ }^{\circ} \mathrm{C}$ 的拉伸应力-应变曲线 与压缩应力-应变曲线如图 $2 a 、 2 b$ 所示。

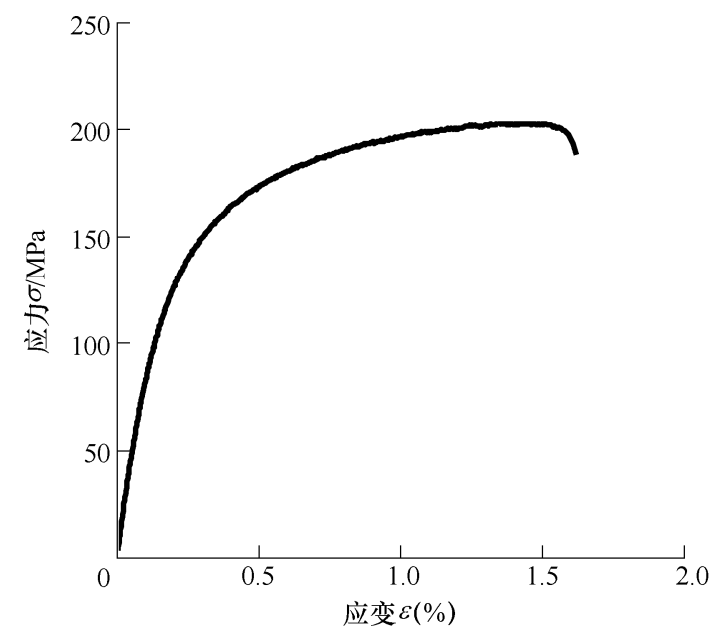

(a) 拉伸应力 - 应变曲线

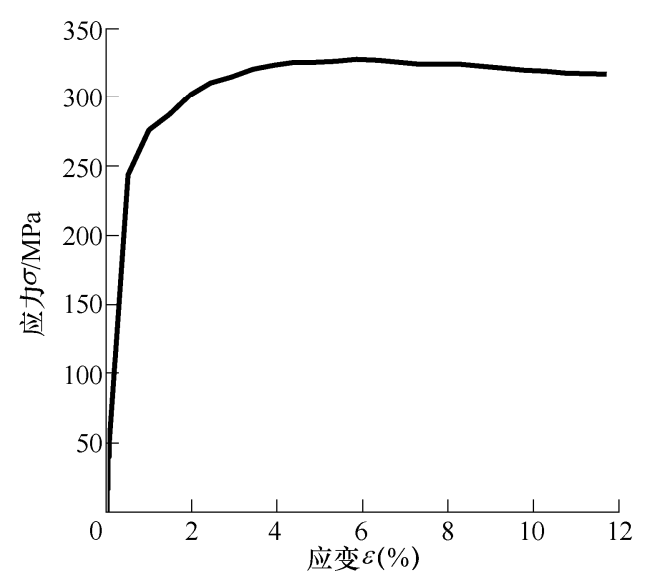

(b) 压缩应力 - 应变曲线 (图中应力、应变取绝对值)

图 2 单调拉伸与压缩应力-应变曲线
从图 2a 拉伸试验可以得出, $500{ }^{\circ} \mathrm{C}$ 下 $\mathrm{HT} 200$ 的延伸率约为 $1.3 \%$, 图 $2 \mathrm{~b}$ 压缩试验可以得出 $500{ }^{\circ} \mathrm{C}$ 时 HT200 的相对压缩率约为 $5.8 \%$ 。在拉伸 和压缩的初始阶段, 应力一应变曲线近似为直线段, 这一阶段材料以弹性变形为主, 随着应变的增加, 应力增长缓慢, 应力-应变曲线不再是直线段, 表明 HT200 在 $500{ }^{\circ} \mathrm{C}$ 下表现出塑性特征。这是因为当温 度在 $0.3 T_{m} \sim 0.7 T_{m}$ 时 ( $T_{m}$ 为金属的熔点), 金属表现 为部分软化 ${ }^{[10]}$, 而灰铸铁的 $T_{m} \approx 1200{ }^{\circ} \mathrm{C}$ 。本文的 试验温度为 $500{ }^{\circ} \mathrm{C}$ 位于在金属的部分软化温度区 间, 并且当温度高于 $0.3 T_{m}$ 时, 非晶机构开始变得 明显, 然后溶解沉淀机构和晶粒边界上的粘性流动 机构等参与作用，同时，像晶粒间脆化和孪生机构 等机构便会消除或几乎消除。此外, 高温时, 剪切 机构甚至是晶块间机构都会大大改变其特性, 它们 的力学现象变得不显著, 并开始清楚地显示出滑移 的扩散特性。这几种现象的总效应就会导致金属塑 性变形能力的提高。

基于试验结果, 通过计算得到真实应力-应变曲 线, $500{ }^{\circ} \mathrm{C}$ 下的单调拉伸、单调压缩结果如表 $1 、 2$ 所示。

表 $1500{ }^{\circ} \mathrm{C}$ 时 HT200 单调拉伸结果

\begin{tabular}{|c|c|c|c|}
\hline 温度 $T /{ }^{\circ} \mathrm{C}$ & $\begin{array}{r}\text { 抗拉强度 } \\
\sigma_{b} / \mathrm{MPa}\end{array}$ & $\begin{array}{c}\text { 弹性模量 } \\
E / \mathrm{GPa}\end{array}$ & $\begin{array}{l}\text { 屈服应力 } \\
\sigma_{s} / \mathrm{MPa}\end{array}$ \\
\hline 500 & 194.76 & 91.20 & 162.8 \\
\hline & \multicolumn{3}{|c|}{$500{ }^{\circ} \mathrm{C}$ 时 HT200 单调压缩结果 } \\
\hline \multirow{2}{*}{ 温度 $T /{ }^{\circ} \mathrm{C}$} & 抗压强度 & 压缩模量 & 屈服应力 \\
\hline & $\sigma_{b} / \mathrm{MPa}$ & $E / \mathrm{GPa}$ & $\sigma_{s} / \mathrm{MPa}$ \\
\hline 500 & 335.45 & 99.77 & 242.0 \\
\hline
\end{tabular}

\section{2 盘式制动器热-结构耦合仿真分析}

基于以上试验获得的力学性能参数, 本文利用 有限元软件 ABAQUS 对盘式制动器在紧急制动工 况下的温度场、等效应力场进行分析。为简化计算 并反映实际制动工况, 对盘式制动器热-结构耦合有 限元模型进行如下假设 ${ }^{[11-12] 。 ~}$

(1) 制动盘和制动块均为各向同性材料。

(2) 不考虑制动过程中制动盘与制动块的磨损 与切削作用。

(3) 制动盘与制动块之间的接触符合库伦摩擦 定律, 且摩擦因数保持不变。

(4) 制动过程中制动盘周围的环境温度保持 不变。

（5）制动过程中仅考虑热传导和热对流，不考 
虑热辐射。

（6）在制动过程中车轮纯滚动, 无抱死情况。

在制动过程中, 制动盘和制动块因摩擦产生大 量热, 其中大部分被制动盘和制动块吸收或通过它 们散发至大气, 因此本文在热-结构耦合分析时, 只 考虑制动盘和制动块, 简化后的盘式制动器有限元 模型如图 3 所示。

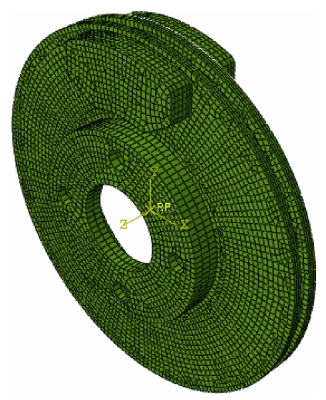

图 3 简化后的盘式制动器有限元模型

\section{1 材料属性的确定}

研究对象是灰铸铁 HT200 制动盘, 金属背板材 料为钢, 摩擦片材料为树脂基体复合材料, 材料参 数如表 $3 、 4^{[13]}$ 所示。

表 3 制动器摩擦副材料特性参数

\begin{tabular}{|c|c|c|c|}
\hline 特性参数 & 制动盘 & 摩擦片 & 背板 \\
\hline 密度 $\rho /\left(\mathrm{kg} / \mathrm{m}^{3}\right)$ & 7220 & 1550 & 7800 \\
\hline $\begin{array}{c}\text { 热传导系数 } \\
\lambda /(\mathrm{W} /(\mathrm{m} \cdot \mathrm{K}))\end{array}$ & 43 & 随温度变化 & 48 \\
\hline $\begin{array}{c}\text { 比热容/C } \\
/(\mathrm{J} /(\mathrm{kg} \cdot \mathrm{K}))\end{array}$ & 503 & 随温度变化 & 452 \\
\hline $\begin{array}{l}\text { 热膨胀系数/ } \\
\alpha /\left(10^{-5} / \mathrm{K}\right)\end{array}$ & 4.39 & 随温度变化 & 1.2 \\
\hline $\begin{array}{c}\text { 弹性模量 } \\
E / \mathrm{GPa}\end{array}$ & 99.77 & 随温度变化 & 209 \\
\hline 泊松比 $v$ & 0.3 & 0.25 & 0.3 \\
\hline
\end{tabular}

表 4 摩擦片随温度变化的特性参数

\begin{tabular}{ccccc}
\hline 特性参数 & \multicolumn{5}{c}{ 温度 $T /{ }^{\circ} \mathrm{C}$} \\
\cline { 2 - 5 } & 20 & 100 & 200 & 300 \\
\hline $\begin{array}{c}\text { 热传导系数 } \\
\lambda /(\mathrm{W} /(\mathrm{m} \cdot \mathrm{K}))\end{array}$ & 0.9 & 1.1 & 1.2 & 1.15 \\
$\begin{array}{c}\text { 比热容 } \\
C /(\mathrm{J} /(\mathrm{kg} \cdot \mathrm{K}))\end{array}$ & 1200 & 1250 & 1295 & 1320 \\
$\begin{array}{c}\text { 热膨胀系数/ } \\
\alpha /\left(10^{-6} / \mathrm{K}\right)\end{array}$ & 10 & 18 & 30 & 32 \\
$\quad$ 弹性模量 & & & & \\
$E / \mathrm{GPa}$ & 2.2 & 1.3 & 0.53 & 0.32 \\
\hline
\end{tabular}

\section{2 摩擦因数和对流换热系数的确定}

摩擦因数 $\mu=0.38^{[14]}$ 。对流换热系数的大小表 征物体与周围空气换热的能力, 由经验公式 ${ }^{[15]}$ 获 得，制动盘表面的平均对流换热系数 $h_{c}$ 为

$$
h_{c}= \begin{cases}0.04\left(k_{a} / d_{0}\right) R e^{0.8} & R e>2.4 \times 10^{5} \\ 0.70\left(k_{a} / d_{0}\right) R e^{0.55} & R e \leqslant 2.4 \times 10^{5}\end{cases}
$$

式中, $k_{a}$ 为空气的热导率; $d_{0}$ 为制动盘外径; $R e$ 为 雷诺数, 其值可由式(2)计算得到

$$
R e=\frac{u_{a} \rho_{a} d_{0}}{\mu_{a}}=\frac{(\omega R) \rho_{a} d_{0}}{\mu_{a}}
$$

式中, $u_{a}$ 为车辆的行驶速度; $\rho_{a}$ 为空气的密度; $\mu_{a}$ 为空气的动力黏度; $\omega$ 为车轮角速度; $R$ 为车轮滚 动半径。

根据相关文献[13]可得： $k_{a}=0.0276 \mathrm{~W} /(\mathrm{m} \cdot \mathrm{K})$; $\rho_{a}=1.13 \mathrm{~kg} / \mathrm{m}^{3} ; \mu_{a}=1.91 \times 10^{-5} \mathrm{~N} \cdot \mathrm{s} / \mathrm{m}^{2}$ 。代入式 (1)、(2)中, 得

$$
h_{c}= \begin{cases}3.50 \omega^{0.8} & \omega>51.81 \\ 7.43 \omega^{0.55} & \omega \leqslant 51.81\end{cases}
$$

紧急制动工况下的车轮角速度 $\omega$ 值 ${ }^{[13]}$, 由式(4) 得到

$$
\omega=76.63-32.33(t+0.125 \exp -8 t-0.125)
$$

基于上述各式, 得到紧急制动工况时的对流换 热系数随时间变化关系如图 4 所示。

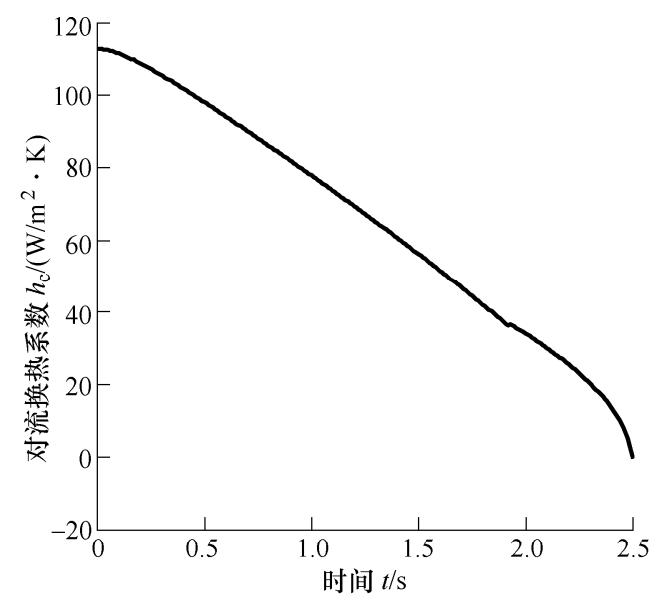

(a)

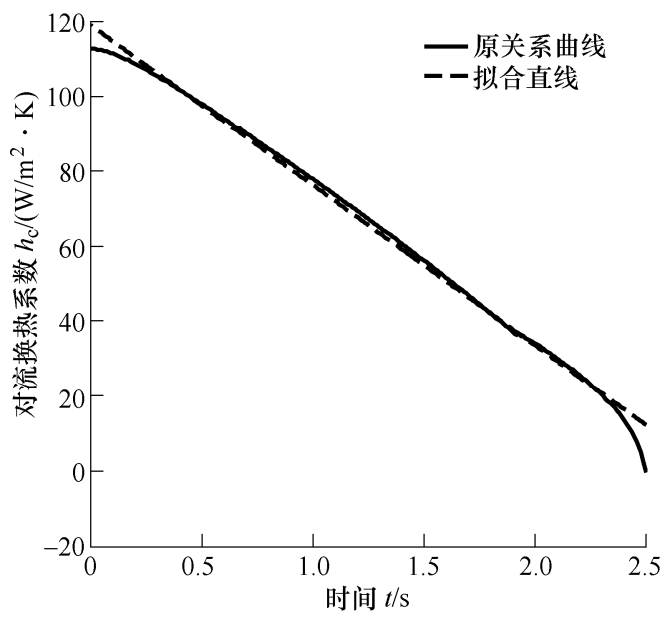

(b)

图 4 对流换热系数随时间变化曲线 
为便于分析, 用一次函数拟合对流换热系数曲 线, 表达式为

$$
h_{c}=-42.7 t+119 \quad 0<t<2.5
$$

对于安装在制动钳中的摩擦块, 其对流换热系数 在制动过程中可认为恒定, 且可按有限空间物体表面 自然对流换热系数计算 ${ }^{[16]}$, 取 $h=5.3 \mathrm{~W} /\left(\mathrm{m}^{2} \cdot \mathrm{K}\right)$ 。

\section{3 载荷及边界条件的确定}

根据制动时的真实情况, 制动块只能沿轴向运 动, 故约束了除 $z$ 轴外的其他 5 个方向的自由度, 制动盘绕 $z$ 轴运动, 约束除轴向旋转方向其他 5 个 方向的自由度, 将参考点与制动盘帽的上下表面做 运动耦合约束。根据工程实际, 紧急制动工况下制 动压力可达到 $4 \mathrm{MPa}$, 因此紧急制动在 $0.2 \mathrm{~s}$ 内 ${ }^{[17]}$ 加载到制动块金属背板上的最大压力为 $4 \mathrm{MPa}$, 汽 车以 $80 \mathrm{~km} / \mathrm{h}$ 的初速度开始制动直至停止 ${ }^{[18]}$, 制动 盘的角速度为汽车的行驶速度与车轮滚动半径的比 值, 因此 $\omega=76.63 \mathrm{rad} / \mathrm{s}$, 制动盘的初始温度假设为 $400{ }^{\circ} \mathrm{C}$ 。

在制动盘上施加的转动惯量应与整车的平动惯 量等效。本文研究所涉及的整车参数如表 5 所示。

\section{表 5 汽车整车相关参数}

\begin{tabular}{lc}
\hline \multicolumn{1}{c}{ 参数名称 } & 参数数值 \\
\hline 车辆满载质量 $M_{0} / \mathrm{kg}$ & 1790 \\
满载时质心高度 $H / \mathrm{mm}$ & 692 \\
轴距 $L / \mathrm{mm}$ & 2725 \\
车辆质心至后轴距离 $L_{b} / \mathrm{mm}$ & 1095 \\
车辆质心至前轴距离 $L_{a} / \mathrm{mm}$ & 1630 \\
轮胎滚动半径 $R / \mathrm{mm}$ & 290 \\
\hline
\end{tabular}

根据《GB12676-1999 汽车制动系统结构、性能 和试验方法》 ${ }^{[18]}$ 中提供的计算方法, 制动盘所施加 的等效转动惯量为

$$
I=\frac{M_{0}\left(L_{b}+0.45 H\right) R^{2}}{2 L}
$$

将表 5 的数值代入式(6)中, 计算得到 $I=38.85 \mathrm{~kg} \cdot \mathrm{m}^{2}$ 。

\section{3 仿真结果分析}

\section{1 紧急制动工况温度场与等效应力场分布}

制动过程中制动盘表面的温度场分布如图 5 所 示。等效应力分布如图 6 所示, 等效应力也称作 Mises 等效应力, 遵循第四强度理论, 反映了制动 过程中热应力与机械应力的综合作用情况。

从图 5 可得出, 制动过程中制动盘表面温度分 布呈非轴对称分布, 制动盘摩擦半径处温度较高, 当 $t=1.563 \mathrm{~s}$ 时, 温度达到最高, 为 $537.3{ }^{\circ} \mathrm{C}$ 。且温
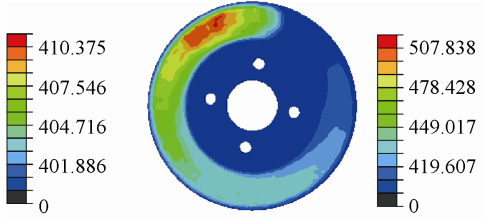

(a) $0.1 \mathrm{~s}$
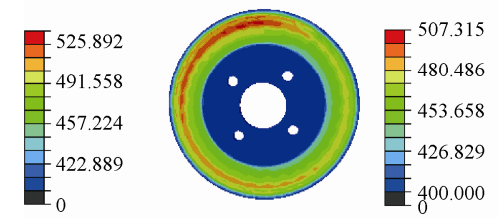

(c) $1.563 \mathrm{~s}$

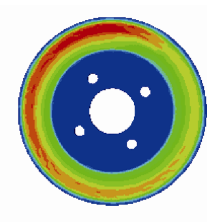

(b) $1.0 \mathrm{~s}$
图 5 紧急制动温度场分布 $\left({ }^{\circ} \mathrm{C}\right)$

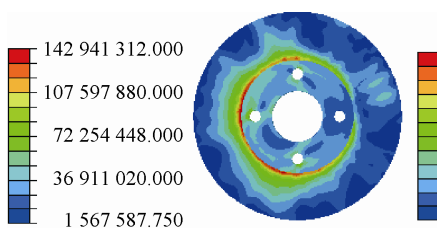

(a) $0.1 \mathrm{~s}$

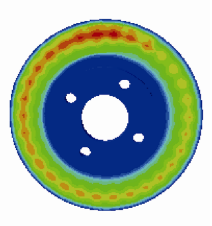

(d) $2.53 \mathrm{~s}$

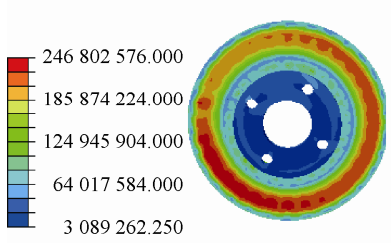

(c) $1.638 \mathrm{~s}$

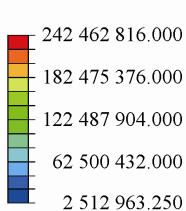

(b) $1.0 \mathrm{~s}$
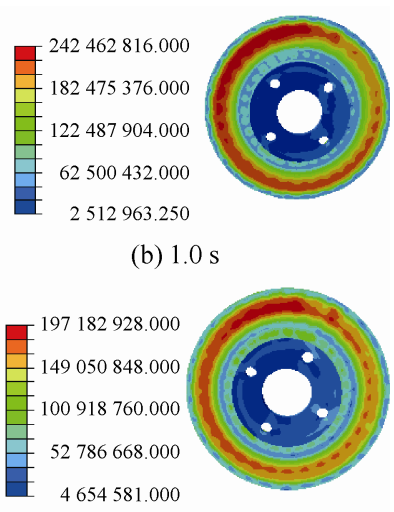

(d) $2.53 \mathrm{~s}$

图 6 紧急制动等效应力场分布 $(\mathrm{Pa})$

度变化趋势是制动初期制动盘表面温度迅速上升, 中期缓慢上升, 后期略有下降。产生这种温度场分 布的原因是, 在制动初期, 摩擦热流的作用远大于 对流散热的作用, 且由于制动初期制动盘的转速较 高, 它与制动块接触产生的热流量更大, 因此制动 初期制动盘表面温度迅速上升, 随着制动的进行, 对流散热的作用逐渐大于摩擦热流的作用, 因此制 动盘的温度开始逐渐下降。

从图 6 可以得出, 制动过程中的等效应力场分 布规律与温度场分布规律相似, 这是因为热应力的 大小与温度梯度成正比。且热应力远大于机械应力, 约为机械应力的 60 倍。当 $t=1.638 \mathrm{~s}$ 时, 等效应力 达到最大, 为 $246.8 \mathrm{MPa}$ 。可以看出最大等效应力 出现的时间比最高温度出现的时间存在一定的滞 后, 这是因为热应力是受热变形受到约束而产生的, 而这需要大量能量, 摩擦热流在制动盘表面产生传 导到制动盘内, 而热传导需要一定的时间, 这就产 生了时间上的滞后现象。

\section{2 制动盘表面温度和周向应力变化规律}

为了进一步分析制动盘表面温度对周向应力的 影响机理, 取图 5 中温度最高点, 其温度与周向应 
力随时间变化曲线如图 7 所示。

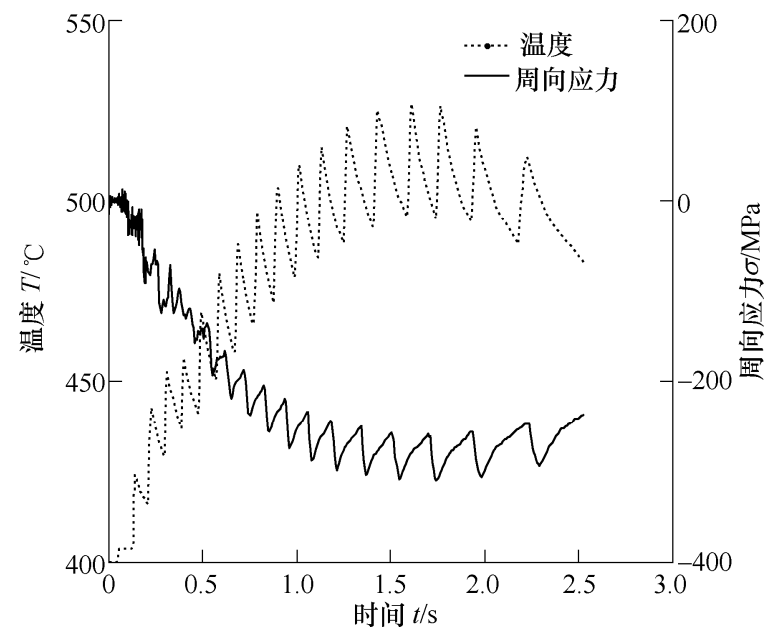

图 7 温度与周向应力随时间变化曲线

从图 7 可以看出, 温度随时间变化曲线呈 “锯 齿状”, 随着制动的进行, “锯齿” 之间的间隔越来 越大, 且节点温度随时间变化曲线呈先增大后减小 的变化趋势。这是因为制动初期制动盘的转速较高, 在摩擦区域内, 单位时间内产生的热量大, 摩擦热 流每次作用在制动盘表面的时间间隔较短, 摩擦热 流作用大于对流换热作用, 导致温度急剧上升, “锯 齿” 的间隔较小。而随着制动的进行, 制动盘的转 速降低, 单位时间内产生的热量变少, 热流作用时 间间隔变长, “锯齿” 的间隔较大。在制动后期, 制 动盘的转速继续降低, 对流换热作用逐渐大于摩擦 热流的产生导致在制动后期温度有所下降。

周向应力随时间的变化规律与温度的变化规律 相似, 这是因为热应力是周向应力的主要组成成分, 而热应力又与温度梯度成正比关系, 相同时间内温 度变化剧烈, 热应力变化大。制动盘与摩擦片接触 区域温度较其他区域较高, 产生的热应力大, 由于 其他结构的约束, 使摩擦区域不能自由膨胀, 因而 产生压应力。

\section{4 热疲劳裂纹内萌生寿命计算}

现有的研究疲劳裂纹萌生寿命的文献均通过等 效应力计算等效应变最终求得应变疲劳寿命的, 但 是这种方法将材料在高温下当作线弹性材料, 没有 对等效应力的拉、压情况做出判断, 同时没有考虑 一次制动过程中的各个应变循环对寿命的影响, 且 等效应力不能准确反映制动盘热裂纹萌生及扩展的 方向, 这些因素必然会导致计算误差。因此利用周 向应变幅根据 Miner 线性累积损伤理论计算一次制 动过程中各个循环造成的损伤, 最终得到应变疲劳
寿命, 并比较考虑和不考虑 HT200 高温塑性这两种 情况下的疲劳寿命。

\section{1 不考虑高温对材料属性影响的热疲劳裂纹萌生 寿命计算}

由前文对紧急制动工况温度场和等效应力场分 布规律可知, 制动盘周向热应力较大, 且根据文献 [19-20]中的试验结果可知制动盘热疲劳裂纹沿径向 分布, 故本文根据应变疲劳公式利用周向应变对制 动盘的热疲劳裂纹萌生寿命进行计算。

在计算疲劳寿命时, 常用的方法有应力疲劳和 应变疲劳, 应变疲劳研究的是大载荷(超过屈服应 力)、短寿命(一般小于 $10^{4}$ )的情况。根据工程经验, 制动盘的热疲劳属于低周疲劳, 故本文采用应变疲 劳方法计算寿命。

根据 Miner 线性累积损伤理论, 在 $k$ 个应变水 平作用下, 各经受 $n_{i}$ 次循环, 则可定义总损伤为

$$
D=\sum_{1}^{k} D_{i}=\sum n_{i} / N_{i} \quad i=1,2, \cdots, k
$$

基于总损伤计算以当前工况制动所能达到的最大的 制动次数, 即为总寿命

$$
N=1 / D
$$

利用 Manson-Coffin 公式描述低周疲劳的应变寿命关系，如式(9)所示

$$
\varepsilon_{a}=\varepsilon_{e a}+\varepsilon_{p a}=\frac{\sigma_{f}^{\prime}}{E}(2 N)^{b}+\varepsilon_{f}^{\prime}(2 N)^{c}
$$

式中, $\sigma_{f}^{\prime}$ 为疲劳强度系数; $\varepsilon_{f}^{\prime}$ 为疲劳延性系数; $b$ 为疲劳强度指数; $C$ 为疲劳延性指数, 根据文献[21] 所述, 利用静态拉伸或压缩试验得到的力学性能参 数估算出这四个疲劳参数。基于前文试验数据, 利 用通用斜率法 ${ }^{[21]}$ 求得这四个参数的值, 如表 6 所示。

表 6 制动盘在 $500{ }^{\circ} \mathrm{C}$ 下的疲劳性能参数

\begin{tabular}{ccccccc}
\hline & 抗压 & 疲劳强度 & 压缩 & 疲劳 & 瘦劳 & 疲劳 \\
材料 & 强度 & 系数 & 模量 & 延性 & 强度 & 延性 \\
& $\sigma_{b} / \mathrm{MPa}$ & $\sigma_{f}^{\prime} / \mathrm{MPa}$ & $E / \mathrm{MPa}$ & 系数 $\varepsilon_{f}^{\prime}$ & 指数 $b$ & 指数 $c$ \\
\hline HT200 & -335.45 & -587.04 & 99770 & -0.09568 & -0.12 & -0.6 \\
\hline
\end{tabular}

忽略 $500{ }^{\circ} \mathrm{C}$ 下 HT200 的塑性特征时, 将其视 为线弹性材料, 一次制动过程中制动盘表面最大周 向应变位置处的周向应变随时间的变化曲线如图 8 所示。根据 Miner 线性累积损伤理论, 利用雨流计 数法 ${ }^{[22]}$ 得到一次制动过程中的各个循环, 求出每一 循环下的总应变幅, 根据式(9)得到每一循环下的寿 命, 并根据式(7)求得每一循环的损伤, 计算过程中 的一些小循环的寿命趋于无限寿命, 所产生的损伤 量很小, 可以忽略, 故本文取应变循环中每一个有 
限寿命循环的损伤如表 7 所示, 最终根据式(8)求出 总寿命。 $N=13409$ 次。

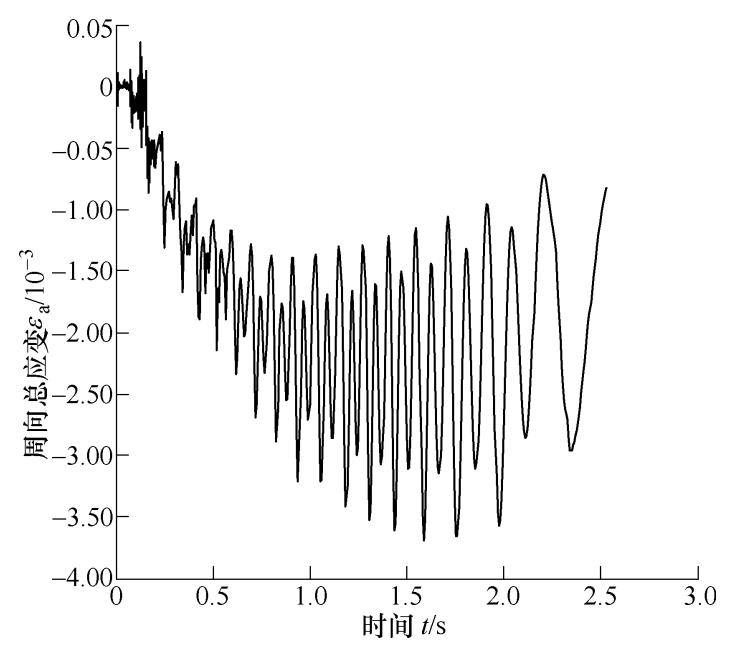

图 8 制动盘表面周向总应变随时间变化曲线

表 7 一次制动过程中各个应变循环的损伤量

\begin{tabular}{|c|c|c|c|c|c|c|c|}
\hline $\begin{array}{l}\text { 应变 } \\
\text { 循环 }\end{array}$ & 1 & 2 & 3 & 4 & 5 & 6 & 7 \\
\hline 损伤 & 1 & 1 & 1 & 1 & 1 & 1 & 1 \\
\hline$D_{i}$ & 18082 & $\overline{908061}$ & 479387 & $\overline{294894}$ & $\overline{193981}$ & 172557 & 573725 \\
\hline
\end{tabular}

表 8 一次制动过程中各个应变循环的损伤量

\begin{tabular}{ccccccccc}
\hline 应变循环 & 1 & 2,3 & 4,5 & 6,7 & 8,9 & 10,11 & $12-14$ & $15-21$ \\
\hline 损伤 $D_{i}$ & $\frac{1}{77020}$ & $\frac{2}{259917}$ & $\frac{2}{226531}$ & $\frac{2}{177624}$ & $\frac{2}{147344}$ & $\frac{2}{131415}$ & $\frac{3}{124975}$ & $\frac{7}{116758}$ \\
\hline
\end{tabular}

求得每一循环的损伤, 如表 8 所示, 表中某些循环 的总应变幅基本一致, 即造成的损伤基本相等, 因 此将损伤相等的循环累加计算, 并最终根据式(8)求 出总寿命。 $N=6514$ 次。

从上述结果可以得出, 考虑高温下 HT200 材料 塑性的影响时, 所获制动盘疲劳裂纹萌生寿命约为 不考虑高温下材料塑性影响时的一半, 当考虑高温 材料塑性特征时, 总应变幅较没有考虑高温下材料 塑性特征时的总应变幅大, 故每次循环下所受的损 伤大, 总体寿命较小。因此在计算制动盘在高温下 的疲劳裂纹萌生寿命时, 应该考虑到高温时的材料 塑性对寿命的影响。

\section{5 结论}

（1）灰铸铁 HT200 虽然属于脆性材料, 但在高 温下显现出明显的塑性特征。

(2) 制动过程中, 制动盘的应力包括机械应力 和热应力, 但以热应力为主, 其数值和对结构的损 伤远大于机械应力; 制动盘工作时的周向应力为压 应力, 其变化同温度的变化规律基本一致。

\section{2 考虑高温对材料属性影响的热疲劳裂纹萌生 寿命计算}

制动盘最大周向应变位置处的周向塑性应变随 时间变化曲线如图 9 所示。利用雨流计数法 ${ }^{[2]}$ 得到 一次制动过程中的各个循环，求出每一循环下的塑 性应变幅, 与弹性应变幅相加, 弹性应变幅为 $\varepsilon_{e a}=\Delta \varepsilon_{e} / 2=\sigma_{s} /(2 E)=-1.213 \times 10^{-3}$, 得到总应变 幅。根据式(9)得到每一循环下的寿命, 并根据式(7)

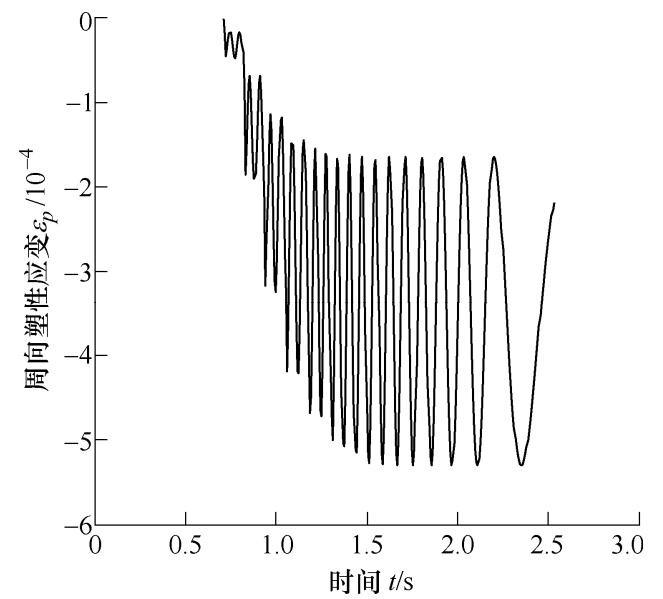

图 9 制动盘表面周向塑性应变随时间变化曲线
（3）高温下灰铸铁 HT200 的塑性特性对制动盘 的疲劳裂纹萌生寿命影响显著, 故预测高温下制动 盘的疲劳裂纹萌生寿命时不可忽略 HT200 的塑性 特性。

根据目前的仿真结果, 常温下单次紧急制动的 温度可达 $200{ }^{\circ} \mathrm{C}$ 左右, 由于实际制动工况的复杂 性, 制动盘温度可能达到 $200{ }^{\circ} \mathrm{C} \sim 500{ }^{\circ} \mathrm{C}$, 甚至更 高。因此, 在研究室温下单次紧急制动或模拟坡道 试验等工况时, 需采用其他温度下的材料特性参数。 后续将进一步对 HT200 在其他温度下的材料特性 进行测试以用于其他工况下制动盘热疲劳裂纹萌生 寿命的预测。

\section{参 考 文 献}

[1] 陈友飞, 李亮, 杨财, 等. 制动器热分析有限差分仿真 模型的研究 [J]. 汽车工程, 2012，34(3): 236-240.

CHEN Youfei, LI Liang, YANG Cai, et al. A study on the finite difference simulation model for brake thermal analysis. [J]. Automotive Engineering, 2012, 34(3): 236-240.

[2] GIGAN G L. Improvement in the brake disc design for 
heavy vehicles by parametric evaluation[J]. Proceedings of the Institution of Mechanical Engineers Part D Journal of Automobile Engineering, 2017, 231(14): 1989-2004.

[3] KAKANDAR E, ROY R, MEHNEN J. A simulation based approach to model design influence on the fatigue life of a vented brake disc[J]. Procedia CIRP, 2017, 59: 41-46.

[4] ZHANG L, YANG Q, WEICHERT D, et al. Simulation and analysis of thermal fatigue based on imperfection model of brake discs[C]/PAMM: Proceedings in Applied Mathematics and Mechanics, 2009, 9(1): 533-534.

[5] SONSINO C M, HANSELKA H. Fatigue life assessment of cast nodular iron disc brakes for railway vehicles [J]. Materialprufung, 2007, 104(11): 562-568.

[6] KIM D J, SEOK C S, KOO J M, et al. Fatigue life assessment for brake disc of railway vehicle[J]. Fatigue \& Fracture of Engineering Materials \& Structures, 2010, 33(1): 37-42.

[7] 王文静, 谢基龙, 李强, 等. SiCp_A356 复合材料制动 盘应力场数值模拟与热疲劳寿命预测 [J]. 机械工程学 报, 2007, 43(6): 127-132.

WANG Wenjing, XIE Jilong, LI Qiang, et al. Stress numerical simulation and thermal fatigue life prediction for SiCp_A356 composite material brake disc[J]. Journal of Mechanical Engineering, 2007, 43(6): 127-132.

[8] 中华人民共和国国家质量监督检验检疫总局, 中国国 家标准化管理委员会. GB/T 228.2-2015 金属材料拉伸 试验第 2 部分: 高温试验方法 [S]. 北京: 中国标准出版 社, 2015 .

General Administration of Quality Supervision, Inspection and Quarantine of the People's Republic of China,

Standardization Administration of the People's Republic of China. GB/T 228.2-2015 Metallic materials-Tensile testing-Part2: Method of text at elevated temperature[S]. Beijing: Standards Press of China, 2015.

[9] 中国航空工业总公司. HB7571-1997 金属高温压缩试 验方法 $[\mathrm{S}]$. 北京: 中国航空工业总公司第三 $\mathrm{O}$ 一研究所 出版, 1997.

Aviation Industry Corporation of China. HB7571-1997 Metallic material-Compressive testing at elevated temperature[S]. Beijing: 301 Institution Press of Aviation Industry Corporation of China, 1997.

[10] 王占学. 塑性加工金属学[M]. 北京: 冶金工业出版社, 2006.

WANG Zhanxue. Plastic processing metallography[M]. Beijing: Metallurgical Industry Press, 2006.

[11] 孟德建, 张立军, 余卓平. 通风盘式制动器热机耦合 理论建模与分析 $[\mathrm{J}]$. 同济大学学报, 2010, 38(6):
890-897.

MENG Dejian, ZHANG Lijun, YU Zhuoping. Theoretical modeling and FEA of therm0-mechanical coupling dynamics of ventilated disc brake[J]. Journal of Tongji University, 2010, 38(6): 890-897.

[12] YEVTUSHENKO A A, ADAMOWICZ A, GRZES P. Three-dimensional FE model for the calculation of temperature of a disc brake at temperature-dependent coefficients of friction $[\mathrm{J}]$. International Communications in Heat \& Mass Transfer, 2013， 42(9): 18-24.

[13] 吴昊. 汽车通风式盘式制动器热一结构耦合分析及结 构的优化设计[D]. 广州: 华南理工大学, 2016 .

WU Hao. Thermal-structure coupled analysis and structural optimization design of vehicle ventilated disc brake[D]. Guangzhou : South China University of Technology, 2016.

[14] 刘献栋, 尚可, 万志帅, 等. 盘式制动器温度模型构建 与温度场仿真 $[\mathrm{J}]$. 汽车工程, 2016, 38(4): 453-458.

LIU Xiandong, SHANG Ke, WAN Zhishuai, et al. Temperature modeling and temperature field simulation for disc brakes[J]. Automotive Engineering, 2016, 38(4): 453-458.

[15] RUDOLF L. Brake design and safety[M]. New York: Society of Automotive Engineers, Inc, 1992.

[16] 杨世铭, 陶文铨. 传热学 [M]. 4 版. 北京: 高等教育出 版社, 2006 .

YANG Shiming, TAO Wenquan. Heat transfer [M]. 4th ed. Beijing: Higher Education Press, 2006.

[17] 方泳龙. 汽车制动理论与设计[M]. 北京：国防工业出 版社, 2005.

FANG Yonglong. Automobile braking theory and design[M]. Beijing: National Defense Industry Press, 2005.

[18］中华人民共和国国家质量监督检验检疫总局. GB126761999: 汽车制动系统结构、性能和试验方法[M]. 北京: 中国标准出版社, 1999.

General Administration of Quality Supervision, Inspection and Quarantine of the People's Republic of China. GB12676-1999: Structure, performance and test method of automobile brake system[M]. Beijing: Standards Press of China, 1999.

[19] 徐济民, 张海泉, 陈强, 等. 快速列车制动盘安全 评定与寿命预测模型 [J]. 清华大学学报, 2006, 46(5): 609-612.

XU Jimin, ZHANG Haiquan, CHEN Qiang, et al. Modeling of safety assessment and useful life time of high -speed passenger car brake discs[J]. Journal of Tsinghua Univ ersity, 2006, 46(5): 609-612.

[20] GIGAN G L, VERNERSSON T, LUNDEN R, et al. Disc 
brakes for heavy vehicles: An experimental study of temperatures and cracks[J]. Proceedings of the Institution of Mechanical Engineers Part D Journal of Automobile Engineering, 2015, 229(6): 684-707.

[21] 姚卫星. 结构疲劳寿命分析[M]. 北京: 国防工业出版 社, 2003.

YAO Weixing. Structural fatigue life analysis[M]. Beijing: National Defense Industry Press, 2003.
[22] SUNDER R, SEETHARAM S A, BHASKARAN T A. Cycle counting for fatigue crack growth analysis[J]. International Journal of Fatigue, 1984, 6(3): 147-156.

作者简介: 刘禹, 男, 1993 年出生。主要研究方向为汽车结构强度与疲劳。 E-mail: yuliu@buaa.edu.cn

刘献栋(通信作者), 男, 1966 年出生, 博士, 教授, 博士研究生导师。 主要研究方向为汽车振动与噪声控制、汽车结构强度与疲劳。

E-mail: liuxiandong@buaa.edu.cn

\section{第 8 届上银优秀机械博士论文奖一佳作奖}

\section{基体表面织构化 TiAIN 涂层刀具的制备与应用的基础研究}

作 者: 张克栋

毕业学校: 山东大学

指导教师: 邓建新

本文将涂层技术与微织构技术相结合, 提出了基体表面织构化 TiAIN 涂层刀具的新概念。通过基体表面织构化, 可改变 基体表面微观结构, 有效增加基体表面的比表面积, 为涂层的涂覆提供良好的附着表面; 另外, 基体表面织构化能够改变 刀-屑界面的接触特征，最终导致涂层刀具在切削过程中的摩擦状态发生显著改善。

利用激光微加工技术进行了 $\mathrm{WC} / \mathrm{Co}$ 基体表面微纳织构的制备, 分析了纳秒和飞秒激光加工参数对所加工微织构和纳织 构表面质量的影响及作用规律, 得到了最佳的微纳织构加工工艺参数。利用真空阴极电弧离子镀技术在织构化基体表面进行 TiAIN 涂层的涂覆, 制备出了三种基体表面织构化 TiAIN 涂层刀具。系统研究了基体表面织构化 TiAIN 涂层的微观结构及物 理机械性能, 并与基体表面无织构的 TiAIN 涂层对比, 提出了基体表面织构化涂层的界面强化机理。通过与 AISI 316 不锈钢 进行球-盘接触式摩擦磨损试验系统研究了基体表面织构化 TiAIN 涂层的摩擦磨损性能。结果表明: 在摩擦过程中, 当涂敷 在光滑表面的 $\mathrm{MoS}_{2}$ 固体润滑剂被完全磨掉时, 摩擦副接触表面之间相互摩擦挤压作用可将储存在微沟槽中的 $\mathrm{MoS}_{2}$ 润滑剂 不断析出补给, 从而在摩擦接触面形成 $\mathrm{MoS}_{2}$ 润滑层; 同时, 微织构可通过扑捉、存储磨损颗粒的作用有效减缓 TiAIN 涂层 的磨损程度。通过车削 AISI 316 不锈钢试验研究了基体表面织构化 TiAIN 涂层刀具时的切削性能, 由试验结果可知基体表面 织构化可显著提高 TiAIN 涂层刀具切削性能: 一方面, 基体表面微织构可促进切削液的渗入, 充分发挥切削液的润滑作用; 同时, 微织构还可起到降低刀-屑接触长度和捕捉磨屑的作用; 另一方面, 基体表面纳织构可显著增加 TiAlN 涂层与基体界 面结合力, 降低由工件材料粘结造成的粘着磨损。 